\title{
Activity Theoretical Analysis and Design Model for Web-Based Experimentation*
}

\author{
Anh Vu Nguyen-Ngoc \\ Department of Computer Science \\ University of Leicester \\ United Kingdom \\ anhvu@mcs.le.ac.uk
}

\begin{abstract}
This paper presents an Activity Theoretical analysis and design model for Web-based experimentation, which is one of the online activities that plays a key role in the development and deployment of flexible learning paradigm. Such learning context is very complex as it requires both synchronous and asynchronous solutions to support different types of interaction, which can take place not only among users but also between the user and the provided experimentation environment, and also between different software components that constitute the environment. The proposed analysis and design model help clarify many concepts needed for the analysis of a Webbased experimentation environment. It also represents an interpretation of Activity Theory in the context of Web-based experimentation.
\end{abstract}

Keywords: Analysis and Design model, Activity Theory, Web-based experimentation.

\section{Introduction}

Since about a decade, several engineering departments in colleges and universities have faced the logistical matters of educating more students with the same resources while maintaining the quality of education. There is also an increasing need to expand the diversity of laboratory resources provided to students. Within this challenging context, the flexible learning paradigm $[1,2]$ could be seen as an appropriate solution. It refers to a hybrid-learning scheme in which the traditional courses are combined with online activities. In engineering education, Web-based experimentation is one of the online activities that plays a key role in the development and deployment of such flexible paradigm. In fact, since the last decade, several institutions have already exploited the usage of the Web infrastructure and developed their experimentation courses in engineering curricula using this medium as a main infrastructure. However, Web-based experimentation is a very complex socio-technical setting [2-4]. As a consequence, understanding the main factors that constitute such particular learning context is an essential step in finding solutions to support and sustain interaction,

* Most of this work has been carried out while the author was with the Ecole Polytechnique

Fédérale de Lausanne (EPFL), Switzerland. 
collaboration and learning processes. Though several Web-based experimentation environments have been developed, such as [5-9], so far, there is still no analysis and design model that is really capture the main characteristics of such learning context, and provide useful guilds for analysts, designers, and developers to design and develop Web-based experimentation environments. This paper proposes such a model.

Section 2 of this paper discusses the major characteristics of Web-based experimentation. Section 3 presents a typical scenario of interaction and collaboration processes in such learning context. The Activity Theoretical analysis and design is discussed in Section 4. Finally, section 5 concludes the paper.

\section{Characteristics of Web-Based Experimentation}

Although there have been several works on Web-based experimentation environment design, development, and deployment, there is still no clear standard for determining the main characteristics of the collaborative hands-on activities in such learning environments. In this section, a list of these essential characteristics is discussed.

\subsection{Hands-On Activities Support}

First of all, the content delivered in engineering courses that rely on Web-based experimentation includes not only static documents, textual presentations, or video presentations but also computation, graphics generated on-the-fly, real devices measurements, and the like. Web-based experimentation can include virtual and/or remote laboratory resources.

In fact, real experimentation is still irreplaceable in engineering curricula since students need to have contact with the apparatus and materials, and that labs should include the possibility of unexpected data occurring as a result of material problems, noise, or other uncontrolled real-world variables. Virtual and remote laboratory resources provide a complement means to carry out real experimentation online and/or at distance. A typical virtual laboratory resource is an interactive experiment that relies on a simulation engine. A typical remote laboratory resource is a physical experimental system that is equipped with the necessary facilities to enable Webbased measuring, monitoring, and manipulation [2].

\subsection{Components Integration}

Due to the complexity of hands-on work [2-4], several components may need to be integrated into the same experimentation environment. These components should help support the whole experimentation process from the preparation stage, to the design stage, to the experiment stage, and to the experimental analysis stage. Each component provides a working space or working console where students carry out some dedicated tasks to solve a particular problem for a complete experiment. Since the output from one stage may serve as the input for the next stages, there should be some linkages between these components. A comparative study have been carried out 
in various engineering courses at the EPFL to determine the most common service spaces that may well require the supporting components for completing typical experimentation assignments $[2,10]$. Each service space can be supported by one or several components developed using different technologies. These spaces are as follows

- The first space that needs to be supported of course relates to the experimentation itself. This can be regarded as the interaction part of the environment. It enables the actual realization of experiments by interacting with virtual laboratory or remote laboratory resources.

- The second space that needs to be supported concerns with tools to carry out interactive design and analysis activities related with the experiment.

- The third space of a Web-based experimentation relates to the collaboration support. This is where the professors and the teaching assistants can interact with the students to monitor their progress and to guild their learning activities; where students interact with each other to get the tasks done.

- Furthermore, a Web-based experimentation environment may also need to integrate some supplementary components, which give access to a number of pieces of information, including relevant reminders or links presenting the underlying theory, experimental protocol, and description of the environment, including the laboratory resources and the environment features that are used in the experiment.

Obviously, depending on the experimental protocol, a Web-based experimentation environment may not need to integrate all of these components.

\subsection{Multi-session Experiment}

Typical Web-based experimentation sessions are mediated by teaching assistants and also by professors responsible for the course. There may be some face-to-face sessions, in which the students work in the laboratory with the presence of the professor and/or teaching assistants, but most of the learning activities take place in flexible sessions. Actually, multi-session experiments are an important factor that helps facilitate students to perform experimentation in a flexible way. In a Web-based experimentation environment, students should be able to carry out several trial-anderror experiments that help them reinforce their understanding of theoretical lectures and physical phenomena in a framework where errors are neither penalized nor hazardous. Ideally, a Web-based experimentation environment should be able to allow students to reconstruct the whole or some parts of the experiment and perform it as many times as they want. Hence, the experimental parameters need to be stored somehow for further reconstruction or reuse of that experiment.

To support multi-session experiments carried out by a single student or by groups of students; many issues need to be addressed, such as the continuity of interaction [11] that allows students to interact smoothly and uninterruptedly with the experimentation environment and the laboratory resources, and also with other students. Several asynchronous and synchronous collaboration facilities need to be considered as well. 


\subsection{Types of Collaboration}

The importance of collaboration among students has been recognized since a long time in education, especially in distance and online education. According to social constructivists, learning is a social construct mediated by language via social interactions [12], where collaboration among learners is a critical concept [13]. In addition, hands-on activities are usually conducted in small groups [2]. Consequently, Web-based experimentation environments should integrate components that help students to actively create their own contextual meaning, rather than passively acquire knowledge structures created by others [3]. These components should facilitate students to interact with their peers, discuss their positions, form arguments, reevaluate their initial positions, and negotiate meaning. Students become responsible for learning as they collaborate with one another, with their environment, and with their teaching assistants and professors. Both synchronous and asynchronous collaborations should be supported in a Web-based experimentation environment.

\subsection{Discretionary of Collaboration}

The autonomy of individual students while working in flexible modalities means that collaboration with other students is, in many cases, not strictly required. In other words, the student can collaborate with other students only when they believe that it is worth to do so. In fact, students participating in the course using the provided Webbased experimentation may enrol in different other courses. This means that they may have different study schedule, and they may carry out different tasks at different times. These variations can make it difficult to find some common times when students can collaborate. As a consequence, even working in groups, students usually work together, either in face-to-face or distance modes, when a due date is approaching, e.g. before the laboratory sessions, or before the laboratory test. Of course, there exist also other modes of group working. Our experience in observing the students' work shows that there are some "well-organized" groups, in which the members clearly divide the tasks for each one. There are also many cases in which only one member of the group does the "whole job". However, depending on the experimental protocol, more precisely speaking, on how the laboratory test is carried out, sometimes it is difficult for the teaching assistants and professors to recognize such problems.

The Web-based experimentation environment should allow students to switch between single working mode and collaborative working mode. This switching should be smooth and transparent as much as possible from the student's point of view.

\section{Typical Scenario of Interaction and Collaboration Process}

Fig. 1 illustrates the interaction and collaboration process happened in Web-based experimentation in which collaborative actors perform a chain of activities to obtain an outcome, i.e. to acquire knowledge from the course (see (1) in the figure). Collaborative actors are, for instance, student groups enrolled in the course and are using the environment to carry out their experimentation. In hands-on sessions, the group size is usually small (consisting of 2 or 3 students) [2,3]. These actors share their common 
background, divide tasks, coordinate their work, and collaborate with each other based on some social rules to get the work done. To support the coordination and communication between these actors, several collaboration and communication facilities may be needed and integrated into the experimentation environment.

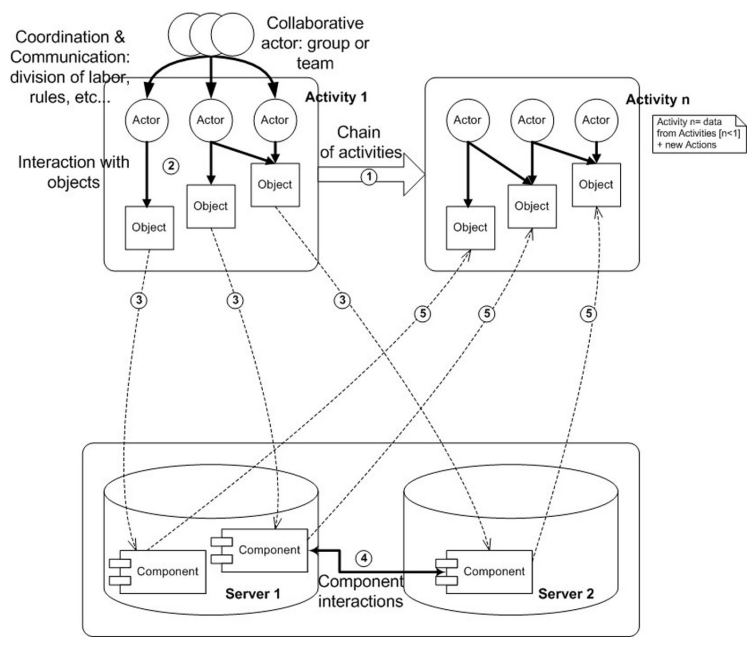

Fig. 1. The interaction and collaboration process of Web-based experimentation

These actors interact with various (software) objects displayed in the GUI of the Web-based environment (2). For example, a student uses the computer mouse to modify the parameters of an electrical drive, which are displayed in the GUI as scrollbars.

These objects are actually the representations of software components (3), which may be located on different servers. The interaction between the actors and the objects may change the status and the behaviours of the components, as well as may invoke the interaction and/or the internal calculating process of these components (4)). In its turn, the interaction between the components at the system level facilitates the interaction process at the user level, which may serve for the next activities of students (5)).

To summarize, this scenario depicts the complexity of the context in which

- Students can collaboratively carry out their hands-on activities in a flexible way.

- The online learning community is heterogeneous and its members may have different roles. The coordination and collaboration among the members of the community may be defined by different social protocols and rules.

- The Web-based experimentation environment itself may integrate a large variety of software components, which constitute what we call the system level. These components are represented by several objects displayed in the interface of the provided experimentation environment.

- The interaction process conducted by the actors, which externally and internally happens in both user and system levels, allow the actors to acquire the outcome for the course. 


\section{Activity Theoretical Analysis and Design}

Obviously, the complexity of Web-based experimentation is caused by several social and technical factors. As a consequent, when studying the collaborative hands-on work in Web-based experimentation, the interaction and collaboration process should be analyzed as a whole, not as any of its constituent entities in separation, since there are close, active, reciprocal, and bidirectional interdependences among these entities.

Actually, the importance of Activity Theory as a framework for conceptualizing human activities has also been studied since a long time by the CSCW and CSCL communities [14, 15]. In an influenced paper published in 1999, Jonassen and RohrerMurphy also argued that Activity Theory has provided a powerful instrument to analyze the needs, tasks, and outcomes for designing constructivist learning environments [16]. They proposed a framework that helps analyze and design a constructivist learning environment. However, one of the most difficult problems for the analysts and designers is how to apply these abstract concepts to a real world problem, e.g. to design a real Web-based experimentation environment that supports online collaborative hands-on activities.

In this section, the Jonassen and Mohrer-Murphy's framework is adapted to introduce a mapping and interpretation from the abstract concepts introduced in Activity Theory into the real context of Web-based experimentation. The constructed framework would help understand and clarify the context of Web-based experimentation from an Activity Theoretical perspective.

\subsection{Activity Theory Concepts}

1. Subject: There could be several types of subjects in the context of Web-based experimentation. Following are the most important ones

a. Professor: is someone who is in charge of the course. His/her role is to design and construct the course pedagogical scenario, to guide students in their learning process during the whole course, and also to evaluate the students' progress and their acquired knowledge.

b. Teaching assistant: is someone who may play a very important role in distributing knowledge in the class. The teaching assistant would help students during hands-on sessions. His/her role could also be to support the course management and administration.

c. Student: the main subject using the environment, who enrols in the course for carrying out experimentation using the environment provided.

d. Technician: is responsible for the configuration of physical equipments in the laboratory.

e. Evaluator, research assistant: is responsible for assessing the effectiveness and efficiency of the environment, and/or proposing further improvement, development, and the like.

2. Object: Different objects can be defined. These different objects are transformed during the course to obtain different outcomes

a. Long-term object: can be composed of both physical and mental products. The physical object could be the deliverables obtained after 
finishing the course, e.g. a course report, or a set of adequate parameters to obtain a stable state of the system. The mental product refers rather to the knowledge, the concepts, or the perceptions of students on a particular engineering domain.

b. Short-term object: objects for each experimental sessions, or modules. Deliverables represented short-term objects could be a report, a mathematical problem to be resolved, a hands-on module to be realized, and the like. Short-term objects can also be the knowledge obtained after finishing these modules.

3. Community: All professors, assistants, students, technicians using the environment for the course form an online learning community, in which the student is the central character and the professors, teaching assistants are usually the central source of knowledge distribution.

4. Rule: Several rules can be defined for a course depending on the course requirements, the laboratory policies, and on the pedagogical scenarios. The task organization among the members of the same groups normally relies on a social protocol or a compromise established within the group or between groups in the community. In hands-on sessions, experimental protocol is what the professors define to guild the students' hands-on steps.

5. Tool, artefact: Tools that need to be integrated should support and reflect the major characteristics of Web-based experimentation as presented in the contextual model. Various tools may be required. The analysts and designers should also consider the question of developing the tools themselves or integrate those having been developed by other institutions.

6. Division of labour: This also means the division of tasks between the members of the learning community. The division of labour is actually dependent upon the learning community and the rules defined for that community.

\subsection{Activity Structure}

This part involves in a definition of the activities that engage the subject. Each activity could be decomposed into its component actions and operations. However, the definition of the activity structure and its granularity is solely based on the pedagogical scenarios as well as on the objectives of the environment evaluators. In a practical course, an activity is usually equated with the task students need to complete [11]. For each activity (or task), actions are the full meaningful experimental steps that need to be realized. Operations are what students do unconsciously by interacting with the environment to complete each step.

In an automatic control laboratory course, for example, a task could be "Modelling and control of an electrical drive". For each task, several actions need to be realized. These actions have an immediate, pre-defined goal, such as "preparing the pre-lab", "manipulating the physical drive", or "analyzing the experimental result". Actions consist of a chain of operations, such as "moving the parameter scrollbar to increase or decrease the value of a parameter of a studying electrical drive". 


\subsection{System Dynamism}

This part investigates the interrelationships between the components that are integrated into the environment. Actually, the interrelationships are dependent upon the pedagogical scenarios defined by the professors. The dynamics of the relationship between members of the community, who use the environment for their learning activities, depends on the social protocol, the division of labour established, and the rules set for the course. Usually, in hands-on sessions, the experimental protocol is pre-defined by the professors and always available for students to follow; hence, for students, the task complexity is mostly dependent upon how they carry out the tasks following the steps defined in the experimental protocol. In addition, the "objectives of work" is also pre-defined, thus collaborative activities are usually not necessarily up to the co-construction level of activity [17].

Fig. 2 summarizes the Activity Theoretical analysis and design model, in which all major elements of Activity Theory are mapped into the context of Web-based experimentation. In other words, the proposed model illustrates our Activity Theoretical vision on the analysis and design of Web-based experimentation environments. Actually, it can also be used as an independent guidance for analysts and designers to analyze and design Web-based experimentation environments.

In fact, this model has facilitated the design and development of the eJournal, which is an electronic laboratory journal integrated into the eMersion experimentation environment. In turn, the iterative design and development of the eMersion environment and the eJournal have validated the reliability and usefulness of the proposed model. The eMersion environment has been used in several automatic control courses offered by the EPFL since several academic semesters. It has also been deployed and tested in other European institutions such as the University of Hanover in Germany, the UNED University in Spain and the Ecole Nationale de Mines St. Etienne in France. More information about the design and evaluation of the eMersion and eJournal could be found in $[2,3,10,18,19]$.

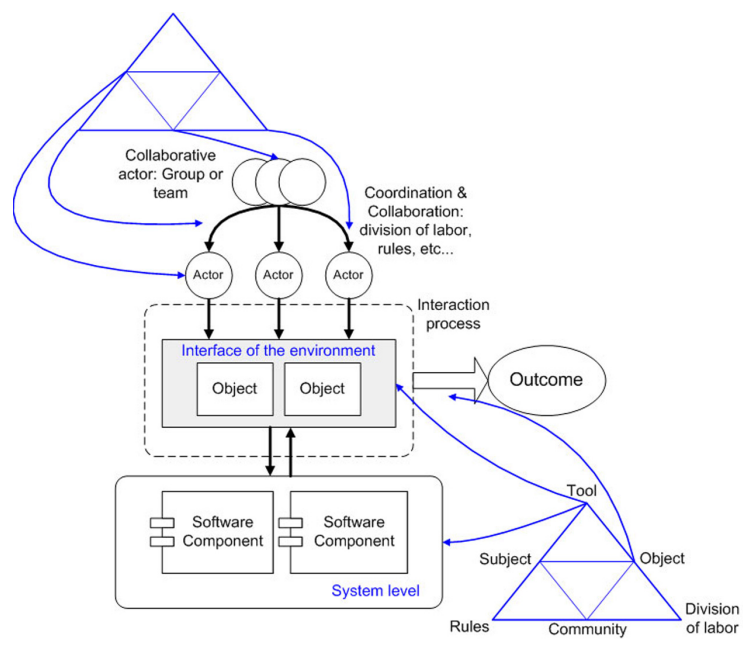

Fig. 2. Activity Theoretical analysis and design model 


\section{Conclusion}

This paper presents what we call Activity Theoretical analysis and design model. It discusses the characteristics of Web-based experimentation and also introduces a typical scenario of interaction and collaboration processes in such learning context. This model shed light on many concepts needed for the design of Web-based experimentation environments. It also represents a mapping from Activity Theory to the context of Web-based experimentation.

The goal of the proposed models is to capture the important aspects concerning the collaborative hands-on activities in a Web-based experimentation environment. The model could be used by a variety of users. Researchers and professors could be based on this model to conduct their study on the students' behaviours and activities in such particular learning context. Environment developers could use the model to facilitate their development tasks as the model focused already on the most relevant issues of the domain. And the developers could use the model to structure the environment in a coherent way.

Acknowledgments. This work would not have been finished without the invaluable support from the eMersion team, EPFL.

\section{References}

1. Holmberg, B.: Theory and practice of distance education, Routledge, London (1995)

2. Gillet, D., et al.: The Cockpit, An effective metaphor for Web-based Experimentation in engineering education. Int. Journal of Engineering Education, 389-397 (2003)

3. Gillet, D., Nguyen-Ngoc, A.V., Rekik, Y.: Collaborative Web-based Experimentation in Flexible engineering education. IEEE Trans on Education, 696-704 (2005)

4. Feisel, L.D., Rosa, A.J.: The role of the laboratory in undergraduate engineering education. ASEE Journal of Engineering Education (2005)

5. Böhne, A., Faltin, N., Wagner, B.: Synchronous tele-tutorial support in a Remote laboratory for process control. In: Aung, W., et al. (eds.) Innovations 2004: World Innovations in Engineering education and research. iNEER in cooperation, pp. 317-329. Begell House Publishers, New York (2004)

6. Schmid, C.: Using the World Wide Web for control engineering education. Journal of Electrical Engineering, 205-214 (1998)

7. Tzafestas, C.S., et al.: Development and evaluation of a virtual and remote laboratory in Robotics. In: Innovations 2005: World innovations in Engineering education and Research. iNEEER in cooperation, pp. 255-270. Begell House Publishers, New York (2005)

8. Ko, C.C. et al.: A Web-based virtual laboratory on a frequency modulation experiment. IEEE Trans on Systems, Man, and Cybernetics, pp. 295-303 (2001)

9. Sepe, R.B., Short, N.: Web-based virtual engineering laboratory (VE-LAB) for collaborative experimentation on a hybrid electric vehicle starter/alternator. IEEE Trans on Industrial Applications (2001)

10. Nguyen-Ngoc, A.V., Rekik, Y., Gillet, D.: Iterative design and evaluation of a Web-based experimentation environment. In: Lambropoulos, N., Zaphiris, P.P. (eds): User-centered design of online learning communities. Idea Group Inc, Pennsylvania, pp. 286-313 (2006) 
11. Nguyen-Ngoc, A.V., Rekik, Y., Gillet, D.: A framework for sustaining the continuity of interaction in Web-based learning environment for engineering education. ED-MEDIA conference, Montreal, Canada (2005)

12. Vygotsky, L.S.: Mind in Society. In: The development of higher psychological processes, Harvard University Press, London (1978)

13. Jonassen, D.H., et al.: Constructivism and computer-mediated communication in distance education. The American Journal of Distance Education, pp. 7-26 (1995)

14. Kuutti, K.: Activity Theory as a potential framework for Human-Computer Interaction research. In: Nardi, B.A. (ed.) Context and Consciousness: Activity theory and Humancomputer interaction, The MIT Press, MA (1995)

15. Nardie, B.A.: Context and consciousness: Activity theory and Human-computer interaction. MIT Press, MA (1996)

16. Jonassen, D.H., Rohrer-Murphy, L.: Activity Theory as a framework for designing constructivist learning environments. Educational Research and Development, pp. 61-79 (1999)

17. Bardram, J.E.: Collaboration, Coordination, and Computer Support: An Activity Theoretical Approach to the Design of CSCW. University of Aarhus (1998)

18. Nguyen-Ngoc, A.V., Gillet, D.S., Sire, S.: Evaluation of a Web-based learning environment for Hands-on experimentation. In: Aung, W., et al. (eds.) Innovations 2004: World Innovations in Engineering education and research. iNEER in cooperation, pp. 303-315. Begell House Publishing, New York (2004)

19. Nguyen-Ngoc, A.V., Gillet, D., Sire, S.: Sustaining collaboration within a learning community in flexible engineering education. In: ED-MEDIA conference. Lugano, Switzerland (2004) 